\title{
PIV study of the effect of piston position on the in-cylinder swirling flow during the scavenging process in large two-stroke marine diesel engines
}

\author{
Journal Article \\ Author(s): \\ Haider, S.; Schnipper, T.; Obeidat, A.; Meyer, K.E.; Okulov, V.L.; Mayer, S.; Walther, Jens Honoré (D) \\ Publication date: \\ 2013-03 \\ Permanent link: \\ https://doi.org/10.3929/ethz-b-000067842
}

Rights / license:

In Copyright - Non-Commercial Use Permitted

Originally published in:

Journal of Marine Science and Technology 18(1), https://doi.org/10.1007/s00773-012-0192-z 


\title{
PIV study of the effect of piston position on the in-cylinder swirling flow during the scavenging process in large two-stroke marine diesel engines
}

\author{
S. Haider $\cdot$ T. Schnipper $\cdot$ A. Obeidat $\cdot$ \\ K. E. Meyer $\cdot$ V. L. Okulov $\cdot$ S. Mayer $\cdot$ \\ J. H. Walther
}

Received: 31 January 2011/Accepted: 14 August 2012/Published online: 11 September 2012

(C) JASNAOE 2012

\begin{abstract}
A simplified model of a low speed large twostroke marine diesel engine cylinder is developed. The effect of piston position on the in-cylinder swirling flow during the scavenging process is studied using the stereoscopic particle image velocimetry technique. The measurements are conducted at different cross-sectional planes along the cylinder length and at piston positions covering the air intake port by $0,25,50$ and $75 \%$. When the intake port is fully open, the tangential velocity profile is similar to a Burgers vortex, whereas the axial velocity has a wakelike profile. Due to internal wall friction, the swirl decays downstream, and the size of the vortex core increases. For increasing port closures, the tangential velocity profile changes from a Burgers vortex to a forced vortex, and the axial velocity changes correspondingly from a wake-like profile to a jet-like profile. For piston position with $75 \%$ intake port closure, the jet-like axial velocity profile at a cross-sectional plane close to the intake port changes back to a wake-like profile at the adjacent downstream cross-sectional plane. This is characteristic of a vortex
\end{abstract}

S. Haider - T. Schnipper - A. Obeidat - K. E. Meyer .

V. L. Okulov · J. H. Walther ( $\square)$

Department of Mechanical Engineering,

Technical University of Denmark,

2800 Kgs. Lyngby, Denmark

e-mail: jhw@mek.dtu.dk

S. Mayer

MAN Diesel \& Turbo, Teglholmsgade 41,

2450 Copenhagen SV, Denmark

J. H. Walther

Chair of Computational Science, ETH Zürich,

8092 Zurich, Switzerland breakdown. The non-dimensional velocity profiles show no significant variation with the variation in Reynolds number.

Keywords Swirl · Scavenging · Two-stroke engine · PIV

\section{Introduction}

Low-speed engines (LSE) are large, two-stroke marine diesel engines that use air swirling inside the engine cylinder for scavenging. The air intake ports are located near the bottomdead-center (BDC). The exhaust port is located in the cylinder head. An exhaust valve is used to open and close the exhaust port, whereas the intake ports are controlled by piston motion. The fresh air enters the cylinder near the bottom-dead-center and flows upward and removes the exhaust gases though the exhaust port at the cylinder head. Since the scavenging air flows from the bottom to the top of the cylinder, this type of scavenging is called uniflow scavenging. Uniflow scavenging accounts for higher engine thermal efficiency due to better air/gas exchange [1]. Efficient scavenging improves the combustion efficiency of the two-stroke diesel engines [2]. The scavenging ports, depending on different designs, are at an angle of $15^{\circ}-25^{\circ}$ with the cylinder radius to impart tangential velocity producing a swirling air column [3]. The resulting in-cylinder confined swirling flow removes the exhaust gases from the cylinder, provides fresh air charge for the next cycle, and introduces swirl to enhance mixing of injected fuel and its consequent combustion. Moreover, it also increases cooling of the cylinder liner, and in case of a non-axisymmetric swirl, can result in an uneven temperature distribution at the walls. Thus investigation and optimization of the scavenging process is a key parameter for the performance and development of fuel-efficient and low-emission marine engines. 
Experimental results available in the scientific literature, focused on studying the uniflow scavenging process in large low-speed marine diesel engines, are very few compared with scavenging in other (smaller and high-speed) two-stroke diesel engines. Previous studies include the experiments by Nishimoto and Takeyuki [4], who obtained the shape of the front surface of the scavenging air using thermocouple in a uniflow model engine. The model engine used hot air for scavenging the cylinder filled with air at room temperature. It was observed that with the increase in the engine RPM (rotations per minute) and port angle, the scavenging air front surface profile changes from jet-like to a wake-like profile analogous to vortex breakdown in an axial flow vortex chamber.

A method was also proposed to obtain a flat profile front surface at an arbitrary Reynolds number for maximum scavenging efficiency. A laser Doppler velocimetry (LDV) experiment was conducted on a model test engine by Dedeoglu [5]. The experiments used a single liquid and cylinder liner with different intake port configurations. The results show that the in-cylinder flow consists of a rotational flow in the cylinder axis region and a potential flow in the near wall region. Nakagawa et al. [2] used twocomponent LDV measurements on a model of a large, low-speed engine with a large-bore acrylic cylinder, and with air as the working fluid. The tangential velocity profile for the piston at TDC was found to depend on the scavenging port angle. Larger port angles resulted in a larger axial velocity drop in the central region of the cylinder, thus occasionally resulting in a reverse flow. Litke [3] studied the influence of the scavenging port angles on the scavenging efficiency by using liquids in a 1:4 scaled engine model. It was observed that the highest scavenging efficiency is obtained with an inlet scavenging port angle of approximately $20^{\circ}$. The results also indicated a better performance of scavenging ports with a combination of different angles compared to the ports with uniform angles.

The large physical size and high in-cylinder pressure for LSE makes experimental investigations in the engine very expensive to conduct, and also present difficult optical access. The scavenging process is unsteady and complex in nature, thus making statistics difficult. In terms of the variation in the geometry of the flow domain, the piston is in continuous motion during the scavenging process, thus changing the cylinder length and also the effective shape of air intake ports. Such complex inlet flow conditions make it difficult to distinguish between inlet effects and swirlingflow effects. Regarding the flow physics, mixing and stratification of exhaust gases with a fresh air charge occurs while the in-cylinder mass flow rate changes between opening and closing of the scavenging ports. This simultaneous variation in flow domain and flow physics consequently affects the in-cylinder swirl characteristics and the type of the vortex generated by the swirl.

Considering the complex nature of the real engine scavenging process, a detailed understanding of the in-cylinder scavenging process requires isolation and consequent study of each flow phenomenon in a simplified form. The complex physics can then be better analyzed by gradually adding complexity. The focus in this study is to characterize the in-cylinder confined swirling flow at different piston positions during the scavenging process. In order to simplify the problem, an experimental down-scale and simplified model of the engine cylinder is developed which is analogous to a straight cylinder connected to a swirl generator but having features like a movable piston, cylinder head, and guide vanes to divert the flow entering the cylinder at a desired angle, etc. In the present study, the cylinder exhaust port is fully open and the exhaust valve is removed. The experimental results will provide a detailed understanding of large two-stroke LSE and fundamental studies in turbulent confined swirling flows.

In the present experiment, air at atmospheric conditions is used as the working fluid and the cylinder length is fixed at $4 D$, i.e., equal to stroke-to-bore ratio for LSE, and where $D$ is the diameter of the cylinder. Stereoscopic particle image velocimetry (PIV) measurements are carried out to study the in-cylinder confined swirling flow. The study does not include the effects of mixing and stratification. The measurements are conducted at four fixed piston positions in translational direction, where the piston partially covers intake ports by 0 (fully open ports), 25, 50 and $75 \%$. PIV data acquisition is performed by keeping the piston at any aforementioned fixed position and then taking measurements at different cross-sectional planes along the length of the cylinder. The piston is then moved to the next position and the procedure is repeated. Conducting measurements at fixed piston positions will give snapshots of the in-cylinder flow characteristics without transient effects induced by the continuous piston motion. This makes the results of the current study very useful for computational studies. In order to study the effect of variation in Reynolds number on the in-cylinder swirling flow, for each given piston position, the measurements are conducted at two different flow Reynolds numbers: $R e=v_{\mathrm{b}} D / v=65,000$ and 32,500 , where $v_{\mathrm{b}}$ denotes the bulk axial velocity in the cylinder, and $v$ the fluid viscosity.

\section{Experimental set-up}

An overview of the experimental set-up is shown in Fig. 1. The scavenging flow test rig is connected with a fan with speed controller and an orifice meter to measure the volumetric flow rate through the set-up. 


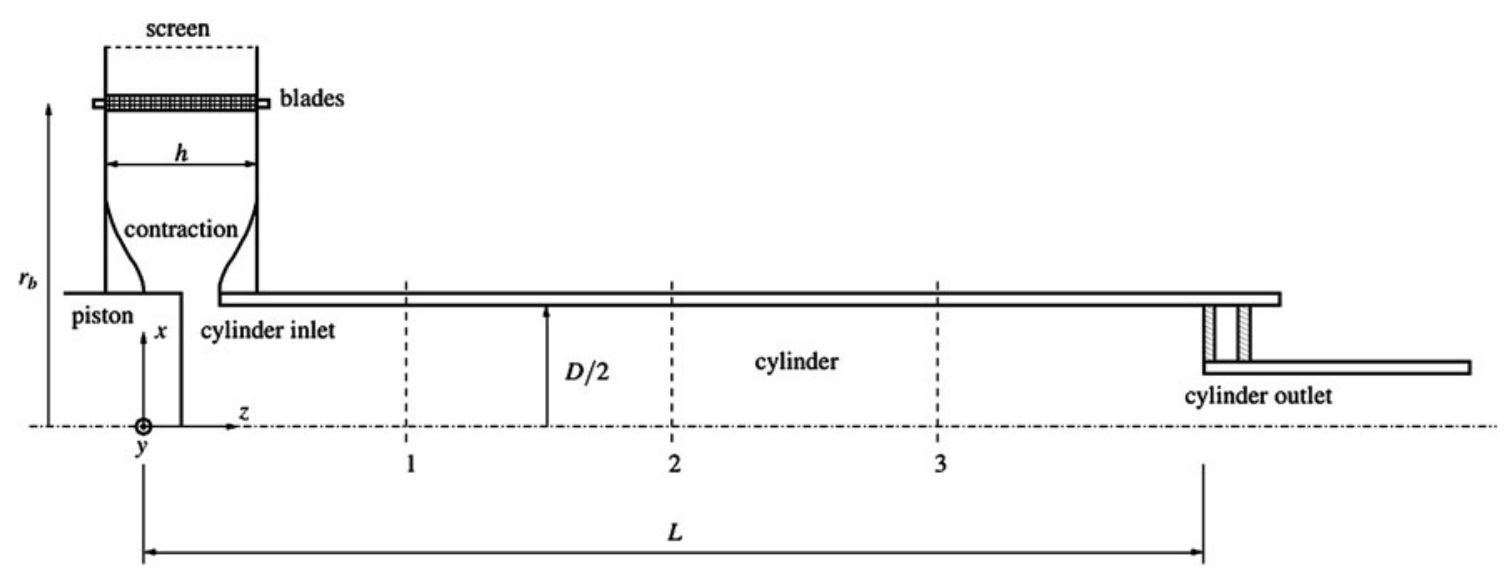

Fig. 1 Schematics of the experimental set-up. The ports are here closed $50 \%$ by the piston. The blades are located at $r_{\mathrm{b}}=250 \mathrm{~mm}$ with a length $h=100 \mathrm{~mm}$. The cylinder has a length $(L)$ to diameter

\subsection{Cylinder}

A transparent acrylic cylinder (produced using a casting process to give good optical properties) is used. The internal diameter $(D)$ of the cylinder is $190 \mathrm{~mm}$, and the total length $L$ of the cylinder is $760 \mathrm{~mm}$. One end of the cylinder enters into the inlet section, and at the other end an outlet section is inserted.

\subsection{Inlet section}

The inlet section (Fig. 1) consists of two transparent ringshaped acrylic plates with an outer diameter of $600 \mathrm{~mm}$. The plates are fitted in parallel by mounting 60 guide vanes in between them, thus serving as walls bounding the flow. The distance $(h)$ between the internal walls of the plates/height of individual blade is $h=100 \mathrm{~mm}$. A screen with $51 \%$ openarea ratio is glued around the outer periphery of the inlet section to obtain a uniform velocity profile at the inlet, cf. Fig. 1. A bell-mouth shape contraction section (Fig. 1), is mounted internally at the inner periphery of the inlet section. The contraction reduced the width of the channel from $100 \mathrm{~mm}$ at the inlet to $40 \mathrm{~mm}$ at the inlet of the cylinder.

An overview of the individual guide vane construction is shown in Fig. 2. Each guide vane has a thickness of $1 \mathrm{~mm}$ and a width of $26.16 \mathrm{~mm}$ (when flat). The guide vanes are then deformed into an arc shape with details given in Fig. 2.

Each individual guide vane is fitted inside the inlet section in a way that one end is aligned in radial direction with the flow entering through the inlet. The other end follows the curved geometry of the guide vane. Thus the flow from inlet enters the guide vane in the radial direction and diverts at an angle of $\alpha_{\text {blade }}=35^{\circ}$ with the radial direction. The guide vanes are mounted at a large radial distance $\left(r_{\mathrm{b}}=250 \mathrm{~mm}\right)$ from the geometric center of the cylinder and close to the inlet (Fig. 1). This will allow
(D) ratio of 4 . The numbers 1,2 , and 3 mark the measurement positions for the PIV system

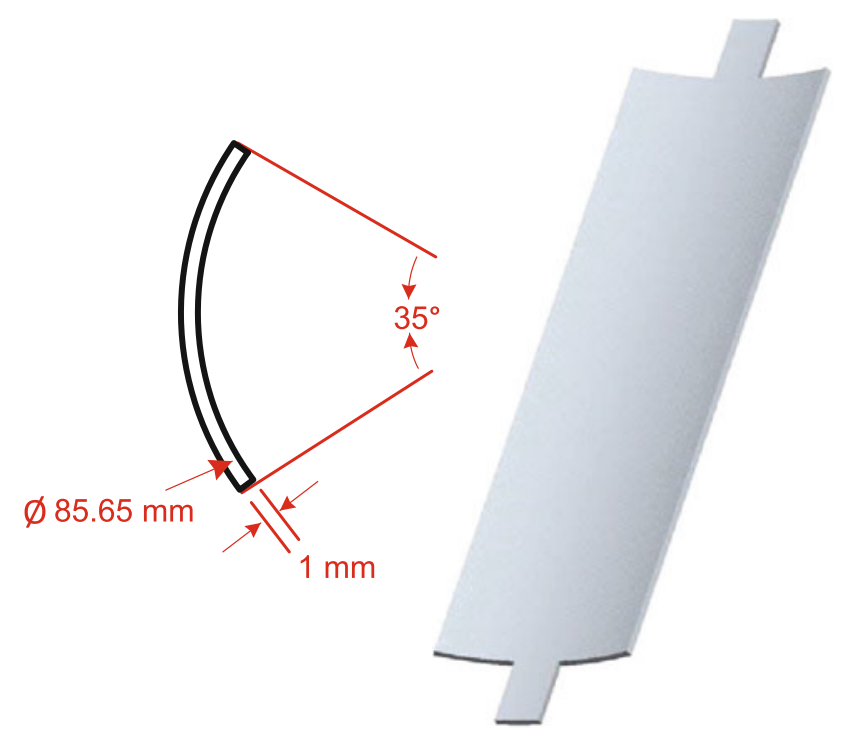

Fig. 2 Illustration of the guide vane blade geometry

some time for the flow to settle after the vanes, thereby minimizing the wake effects behinds the guide vanes. Further, before entering the cylinder, the flow enters the contraction section, which will accelerate the flow and reduce the velocity fluctuations. The acrylic cylinder is entered into the inlet section from one side, and from the other side a transparent piston is mounted. The piston can slide inside the cylinder, and thus partially or fully close the cylinder inlet section, similar to the way that in real engines the reciprocating piston uncovers and covers the scavenging ports. However, the outer diameter of the piston is larger (by the wall thickness) than the cylinder inner diameter. This limits the displacements of the piston to a position where it fully covers the cylinder intake port. The current work is focused on studying the in-cylinder swirling flow when the piston is at the BDC (the inlet to the 
cylinder is fully open) and piston positions where it covers the intake port by 25,50 and $75 \%$.

\subsection{Outlet section}

The outlet section consists of a smaller internal diameter pipe $(d=110 \mathrm{~mm})$ and length $(l=1415 \mathrm{~mm})$. The large length of the pipe will minimize any effect on the nature of the swirling flow inside the cylinder due to bending in the connecting pipes to the orifice plate and fan. The shape of the outlet provides a flat-bottom head to the cylinder. The outlet section can slide inside the cylinder and facilitates experiments at a desired effective length of the cylinder, which in this study is kept fixed at $4 D$.

\section{Stereoscopic PIV setup}

An overview of the stereoscopic particle image velocimetry set-up is given in Fig. 3. The laser optics and the two cameras are mounted on a large traverse system that moves in the axial direction, i.e., along the length of the test cylinder. The traverse is carefully aligned with the cylinder and uses a stepping motor for accurate positioning. The traverse system moves independently of the test rig.

\subsection{Alignment and calibration}

A NewWave Solo Nd:Yag pulse laser (120 mJ pulses at wavelength $532 \mathrm{~nm}$ ) is mounted on the traverse so that the laser sheet is exactly perpendicular to the cylinder axis. The approximate laser sheet thickness is $4 \mathrm{~mm}$. Two Dantec HiSense cameras with $1,344 \times 1,024$ pixels and pixel size of $6.45 \mu \mathrm{m}$ are equipped with $60 \mathrm{~mm}$ focal length lenses and green light filters. Both cameras are in Scheimpflug condition and mounted on the same traverse as the laser source. The cameras are at one side of the laser sheet, and the cylinder is placed between the two cameras (Fig. 3). The calibration target is kept on a disc of same diameter as the internal diameter of the cylinder and slides inside the test cylinder. The calibration target is kept aligned with the laser sheet at a given measuring cross-sectional position. Both cameras are aligned in a way that their rectangular frames capture the maximum area of the given cross-section within the pipe diameter, thus avoiding measurements close to the cylinder wall due to strong reflections of the laser light. The calibration is performed at a single measuring position. Dantec DynamicStudio software is used for PIV measurements and data processing. Calibration is performed using the 3rd order xyz-polynomial imaging model which is capable of handling distortion caused by the lens or curved windows [6]. In order to account for the possible misalignment of the calibration target with the laser sheet, disparity error correction is performed. Since the relative positions of the laser source and the two cameras are fixed, the test cylinder is considered axially aligned and the same calibration is used for all the other measuring cross-sections.

The F-number is set to 8 for the camera receiving the forward scatter and for the camera receiving the backward scatter, the F-number is set to 4 .
Fig. 3 Illustration of the stereoscopic PIV set-up

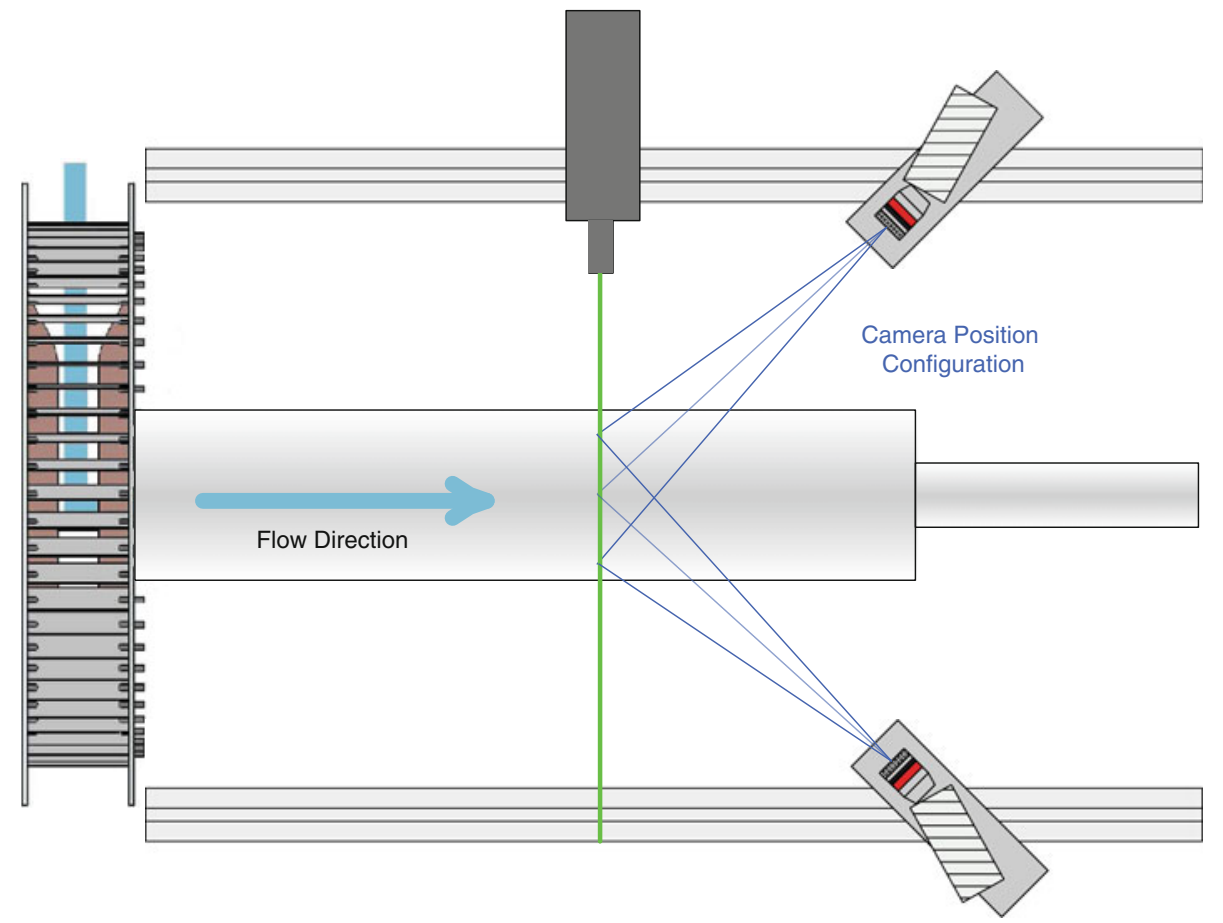




\subsection{Seeding}

The seeding generator contains a $75 / 25 \%$ by volume glycerol-water solution, and the size distribution of seeding droplets is in the range of 1-3 $1 \mathrm{~m}$. For a uniform and adequate seeding, the particles should mix properly with the incoming air entering the inlet section. For this purpose, a metal frame with a diameter of $860 \mathrm{~mm}$ and width $150 \mathrm{~mm}$ is mounted around the inlet section.

A plastic pipe with a diameter of $40 \mathrm{~mm}$ is tied with the internal periphery of the metal frame in a helical shape so as to cover the whole breadth of the metal frame and, consequently, the inlet surface. A large number of holes (approximately $1.5 \mathrm{~cm}^{-2}$ with a diameter of $3 \mathrm{~mm}$ ) are drilled in the pipe wall facing the inlet of the experimental set-up, and the two ends of the pipe are connected to the seed generator. The radial distance of the pipe wall from the inlet surface is sufficiently large to minimize any effect to the incoming flow and provide a uniform seeding across the inlet surface.

\section{Data acquisition}

The measurements are conducted at different cross-sectional planes along the length of the cylinder, with their distances measured from $z=0$ as reference, i.e., the piston surface when the port is fully open (cf. Fig. 1). For every position, a minimum of 994 PIV snapshots are taken. Data processing and analysis of the PIV images is performed using multi-pass "Adaptive Correlation" algorithm in Dantec DynamicStudio software. The initial interrogation window size is $128 \times 128$ pixels, with two refinement steps to a final interrogation area of size $32 \times 32$ pixels and a $50 \%$ overlap of the side of the interrogation area. The time-between-pulses (TBP) was optimized by testing several values. The largest value that gave a low amount of outlier vectors was selected. This was performed for each combination of measurement position, piston position, and Reynolds number.

\section{Results and discussion}

In order to present the results in a comprehensive manner, the results for the case of fully open intake port are presented and discussed first. The results for the cases with 25 , 50 and $75 \%$ partially closed intake port are then discussed in a successive manner. The measurements are conducted for two flow Reynolds numbers: $R e=v_{\mathrm{b}} D / v=65,000$ and 32,500. The results are presented for Reynolds number 65,000 , since no significant differences are observed compared to Reynolds number 32,500 for a given measuring plane and piston position.
A significant effort has been made to make the experimental set-up rotationally symmetric. In order to assess the uncertainties involved in the measurement process and the repeatability of the experimental results, different measurement sessions were conducted for a given position and Reynolds number. These measurement sessions were conducted on different days, dismantling and reassembling of the PIV set-up (Fig. 3) between tests and using different TBP each time. The results from different measurement sessions are labeled in each presented profile and represent repeated experiments, but with different TBP for PIV and on a reassembled PIV set-up. The profiles of the tangential and axial velocity components include the error bars. The error bars are based on standard error (SE) of the mean, with upper and lower $95 \%$ confidence limits, and they present the uncertainty involved in a given measurement

$$
\mathrm{SE}=\frac{\sigma}{\sqrt{n}},
$$

where $\sigma$ is the standard deviation of all the observations for a given measurement, and $n$ represents the total number of observations.

The degree of swirl in a given swirling flow is quantified by the non-dimensional swirl number $S$. The swirl number in this experiment is measured using the relation for the design swirl parameter given by Gupta et al. [7] and Alekseenko et al. [8]

$S \equiv \frac{2 F_{\theta, z}}{F_{z, z} D}$,

where $F_{\theta, z}$ and $F_{z, z}$ denote the flux of angular and axial momentum in the flow direction. The angular momentum is estimated from the radial flux of angular momentum

$F_{\theta, z} \approx F_{\theta, r}=\rho v_{\theta} v_{r} r(2 \pi r h)$,

where $h$ denotes the height of the channel at the radius $r$ (Fig. 1), and

$F_{z, z}=\rho v_{\mathrm{b}}^{2}\left(\frac{\pi}{4} D^{2}\right)$,

where $v_{r}=v_{r}(r)$ is related to the bulk velocity $v_{\mathrm{b}}=v_{r} 8 r h D^{-2}$. Thus, the swirl number is

$S=\frac{v_{\theta}}{v_{r}} \frac{D}{4 h}$.

At the guide vanes $(r=250 \mathrm{~mm})$ the velocity ratio $v_{\theta} v_{r}^{-1} \approx \tan \left(\alpha_{\text {blade }}\right)$, and hence the design swirl number is $S=0.33$. In addition, preliminary laser Doppler anemometry (LDA) experiments have been conducted to study the wake behind the blades (not shown). At $r=200 \mathrm{~mm}$, the measured flow angle is $26^{\circ}$, which results in an effective swirl number of $S=0.23$. 


\subsection{Fully open ports}

When the intake port is fully open, the in-cylinder swirling flow results in a high velocity region at an intermediate radial position between the cylinder wall and geometric center cf. Fig. 4a). Here $R$ is the radius of the cylinder. $X$ and $Y$, shown in Fig. 4, represent the $x$-axis and $y$-axis, respectively, when the origin of the coordinate system is defined as the cylinder axis. The origin of the $z$ axis is at the piston surface when the port is fully open. Contour color represents the axial velocity (out of the plane component) $v_{z}$ normalized by bulk flow velocity $v_{\mathrm{b}}$. It can be seen that the resulting in-cylinder swirling flow is comprised of a concentrated vortex with a core surrounded by a high-velocity region. The velocity magnitude in the vortex core is very low compared to its surroundings. A low-velocity region exists at a larger radial positions close to the cylinder wall (not shown). Although great care has been taken to make the experimental set-up rotationally symmetric, the recirculating vortex core is asymmetric, i.e., the mean vortex position is not coinciding with the geometric center of the cylinder.

Figure 5a shows that when the intake port is fully open, the tangential velocity profile at $z / D=0.963$ is comprised of a forced vortex region (solid body rotation) and an outer region with very low rotation or weak vorticity. The $X_{\mathrm{v}}$ shown in Fig. 5a represents the $x$-axis when the origin of the coordinate system is shifted to the mean vortex center. Higher velocities are observed in the radial position where the inner forced and outer free vortices meet. The vortex core region has low-velocity magnitude compared to other regions of the cylinder (except near the cylinder wall, as in the current experiment measurements near the wall have not been taken).

The axial velocity $v_{z}$ has a wake-like profile (cf. Fig. 5b) and has an overall magnitude higher than $v_{\theta}$. This shows that flow has low swirl intensity. The size of the vortex core increases downstream as both the tangential and axial velocities gradually decay downstream of the cylinder, and the velocity peaks become less distinct (Fig. 5d, e, g, h).

The flow at $z / D=0.963$ has a large negative value of the normalized mean axial vorticity $\left(\omega_{z}\right)$ in the core region (calculated by first normalizing $x, y$ with cylinder radius $R$ and $u, v$ with bulk flow velocity $v_{\mathrm{b}}$, cf. Fig. 5c). The vorticity distribution appears to have a Gaussian-like profile. The vorticity is concentrated in a small tubular region near the vortex center and decays rapidly outward in the radial direction, indicating a concentrated vortex profile (cf. [9]). Very low vorticity is observed only at large radial distances $X / R=0.5$ from the vortex center.

With the decay of the swirl along the cylinder length, the vortex core gradually loses its vorticity. The size of the core increases, and the weak-vortical region diminishes downstream of the cylinder (Fig. 5f, i).
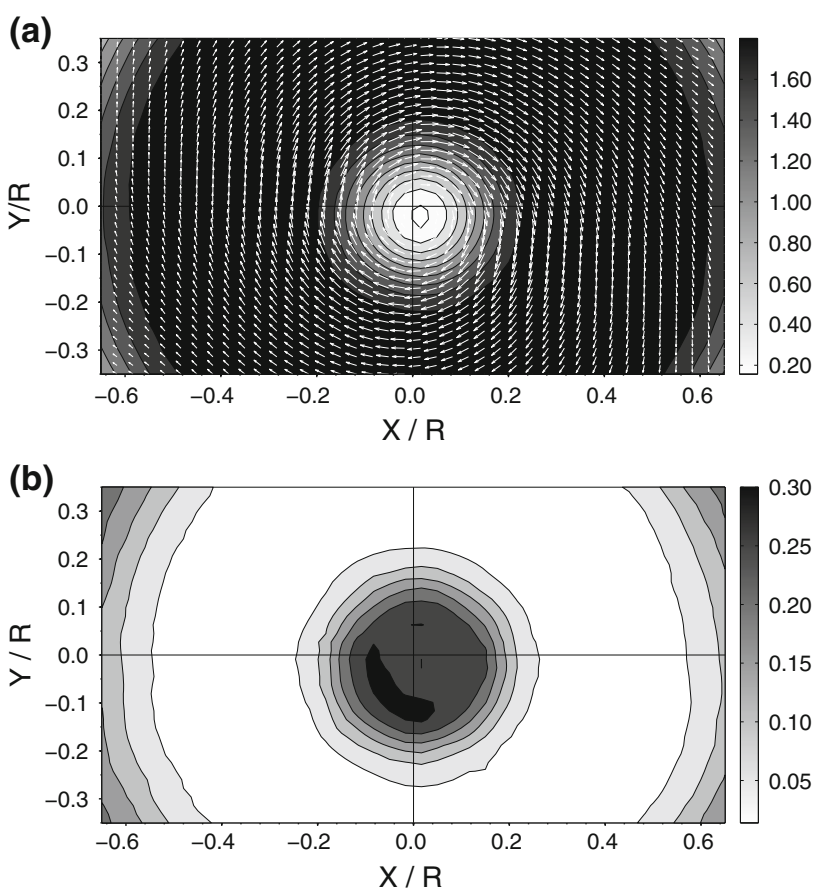

Fig. 4 Time average flow fields at $z / D=0.963$ with fully open ports: a normalized velocity field (contour colors represent magnitude of out-of-plane velocity component); b normalized turbulent kinetic energy

At $z / D=0.963$, the vortex core exhibits an asymmetric distribution of the mean turbulent kinetic energy (TKE), cf. Fig. 4b, indicating that the flow is three-dimensional. The largest value of TKE is observed in the vortex core region. However, the kidney shape of the region with high variance indicates the vortex precession superimposed on the threedimensional velocity variations. The region outside the vortex core has very low turbulent kinetic energy, except that beyond a half-cylinder radius TKE starts increasing again towards the cylinder wall region. This is due to the $90^{\circ}$ bend between the swirl generator outlet and the test cylinder (Fig. 1). The flow separates at the wall while entering the cylinder, which increases the turbulence. As the swirl decays downstream (not shown), the overall magnitude of the turbulent kinetic energy also decreases, but its spatial distribution increases due to enlargement of the vortex core and comparatively more uniform velocity distribution.

\subsection{Ports closed by $25 \%$}

As the piston is translated to the positions partially covering the intake port, the in-cylinder flow becomes comparatively more chaotic. The possible reason is that the piston constitute a bluff body with a sharp edge to the flow entering the cylinder and the port area also reduces. This produces increased velocity fluctuations at the inlet 

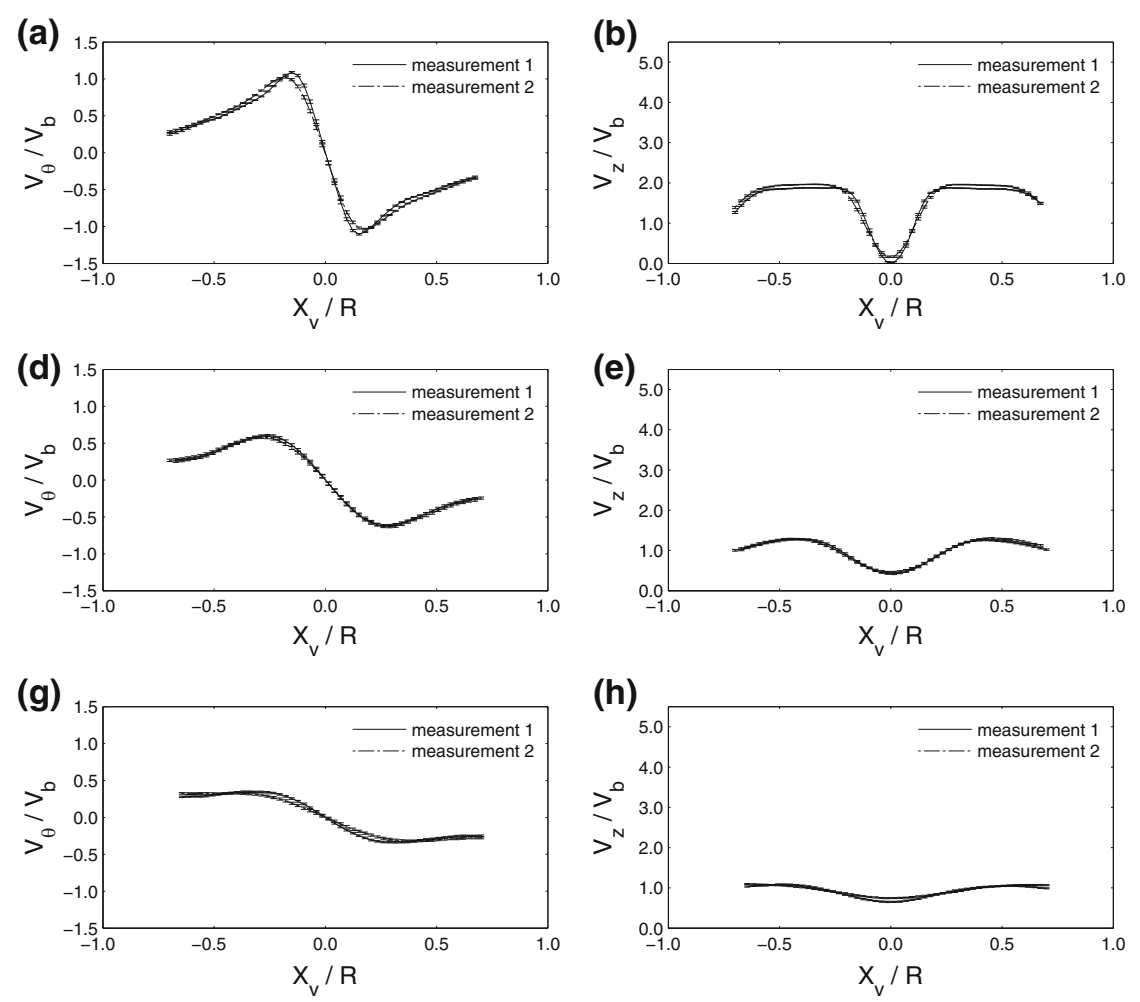
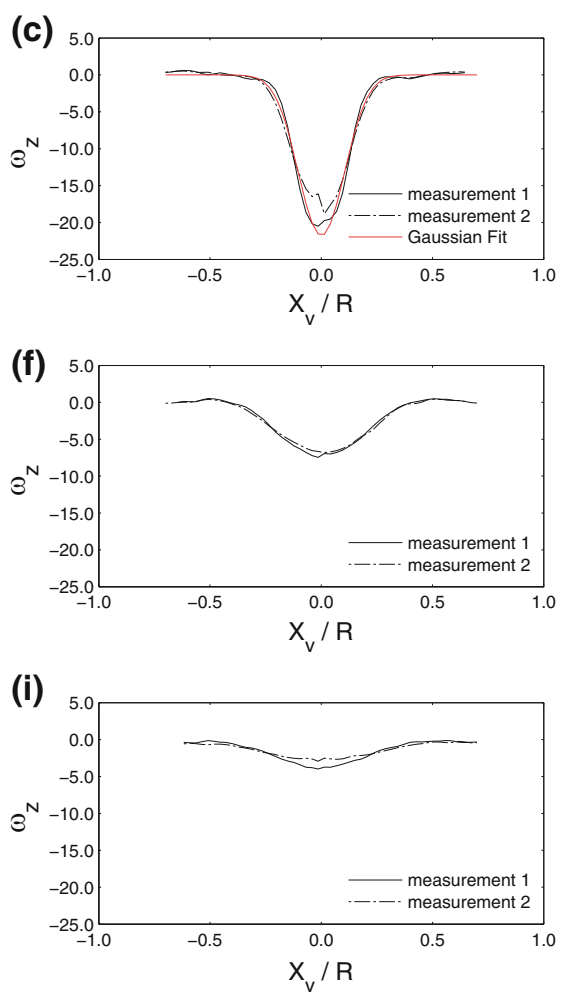

Fig. 5 Normalized time averaged profiles with fully open ports: a tangential velocity profile at $z / D=0.963 ; \mathbf{b}$ axial velocity profile at $z / D=0.963$; c axial vorticity profile at $z / D=0.963$; d tangential velocity profile at $z / D=2.016 ;$ e axial velocity profile at

compared to when the port is fully open and affects the incylinder velocity distribution.

At $25 \%$ partially closed port (Fig. 6a, d, g), the maximum value of $v_{\theta}$ at $z / D=0.963$ has decreased compared to fully open port. The profile shows a less distinct peak at the interface of outer region and inner enlarged forced vortex region. The size of the forced vortex region increases downstream and at $z / D=3.068$, the overall $v_{\theta}$ profile changes to a forced vortex i.e. the high $v_{\theta}$ region shifts to near wall position and eliminating the outer free vortex region. The overall $v_{\theta}$ magnitude decreases at all measuring positions compared to fully open port. The $v_{z}$ at the near outlet positions changes from wake-like profile to a more uniform distribution cf. Fig. 6b, e, h. At position $z / D=$ 0.963 near the inlet, the peak values of $v_{z}$ magnitude is higher than its value at the open port position of the piston.

Figure $6 \mathrm{c}, \mathrm{f}, \mathrm{i}$ shows the mean axial vorticity for the positions $z / D=0.963, z / D=2.016$, and $z / D=3.068$, respectively. Compared to the fully open port case, the overall vorticity magnitude is reduced. At $z / D=0.963$, similar to the fully open port, the vortex core region has the strongest vorticity. However, the maximum vorticity magnitude in the vortex core region is less than half the value observed in the case of fully open intake port. Due to the increase in the size of the vortex core region, the spatial $z / D=2.016 ; \mathbf{f}$ axial vorticity profile at $z / D=2.016 ; \mathbf{g}$ tangential velocity profile at $z / D=3.068 ; \mathbf{h}$ axial velocity profile at $z / D=3.068 ; \mathbf{i}$ axial vorticity profile at $z / D=3.068$

distribution of the vorticity around the vortex center has also increased. This indicates that the Burgers vortex profile is diminished in such a way that the vorticity from strong vortical region (vortex core) is transferred to the surrounding weak vortical regions, and thus the vorticity of the outer (free vortex) region increases. With the flow downstream, the vorticity distribution tends to become more uniform at $z / D=2.016$, and at $z / D=3.068$ a uniform vorticity distribution is observed, cf. Fig. 6f, i. The fluctuations in the vorticity profile plots are due to measurement noise.

At $z / D=0.963$ the maximum value of the turbulent kinetic energy seems to have increased when the piston partially closes the intake port by $25 \%$. The maximum value of TKE is still observed in the vortex core region, however the region with low value of TKE, observed in the case of fully open port, has shrunk, thus indicating an increase in the value of TKE from the half-cylinder radius to the regions close to the wall (Fig. 9a).

\subsection{Ports closed by $50 \%$}

With the piston covering $50 \%$ of the intake port, the asymmetry in the distribution of $v_{\theta}$ at $z / D=0.963$ increases (cf. Fig. 7a.) 

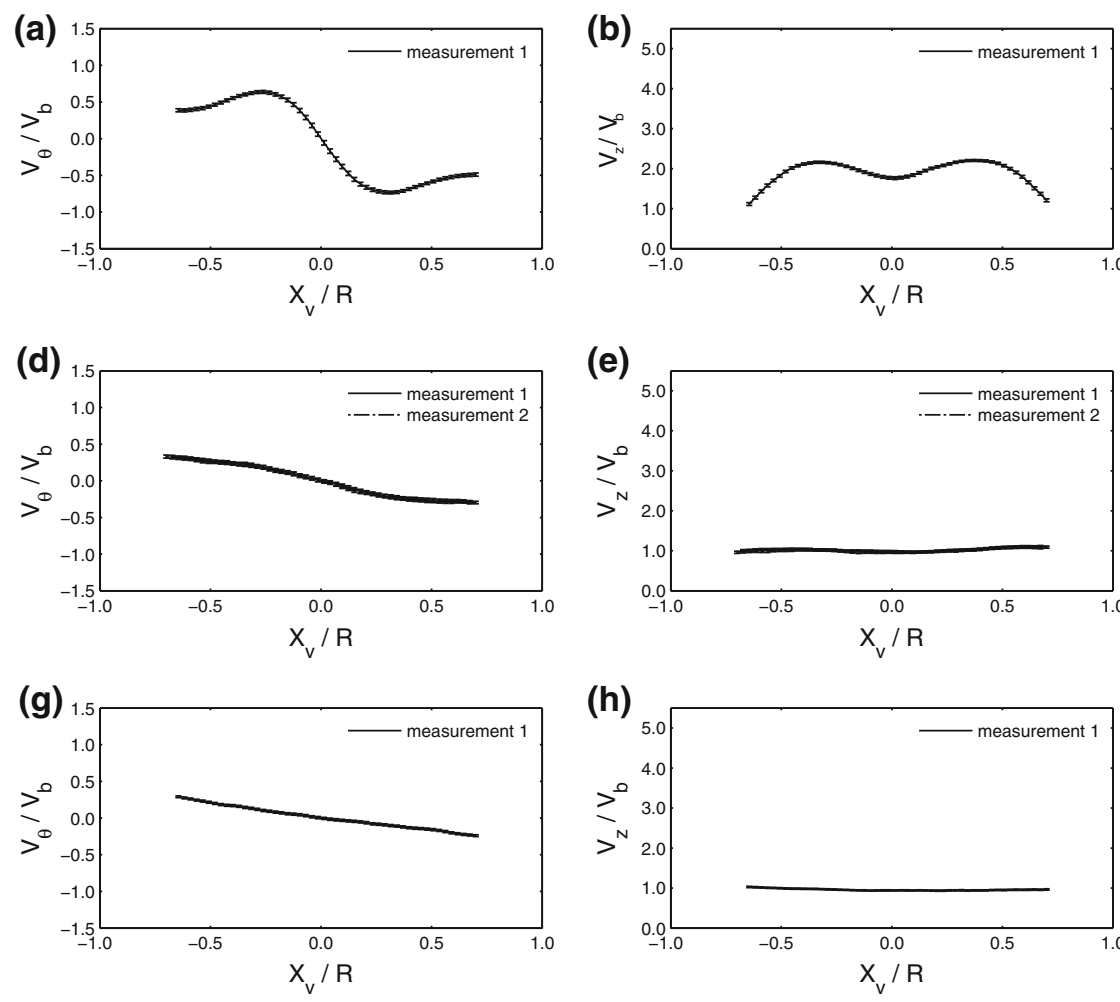

(e)

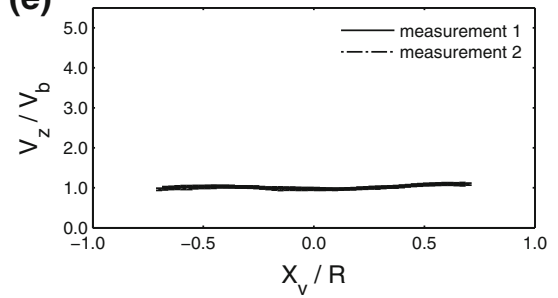

(h)

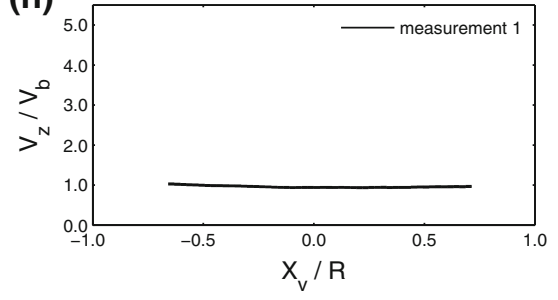

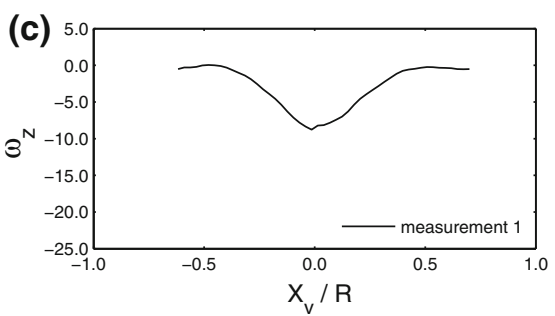

(f)
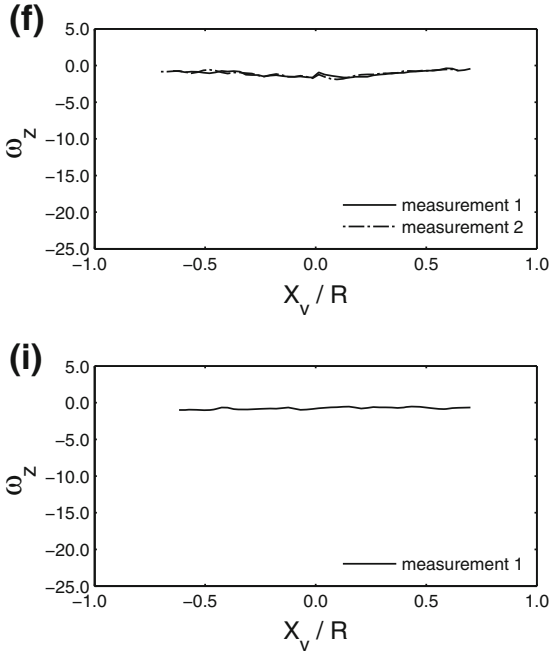

Fig. 6 Normalized time averaged profiles with $25 \%$ closed ports: a tangential velocity profile at $z / D=0.963 ; \mathbf{b}$ axial velocity profile at $z / D=0.963$; c axial vorticity profile at $z / D=0.963$; d tangential velocity profile at $z / D=2.016 ;$ e axial velocity profile at $z / D=2.016 ; \mathbf{f}$ axial vorticity profile at $z / D=2.016 ; \mathbf{g}$ tangential velocity profile at $z / D=3.068 ; \mathbf{h}$ axial velocity profile at $z / D=3.068 ; \mathbf{i}$ axial vorticity profile at $z / D=3.068$

towards the wall (Fig. 7b). However, there is a rapid decay in the velocity peaks from position $z / D=0.963$ to $z / D=$ 2.016 and again at $z / D=3.068$, where a uniform distribution of $v_{z}$ is observed (cf. Fig. 7e, h).

For $50 \%$ intake port closure, the mean axial vorticity at $z / D=0.963$ is shown in Fig. 7c. The ripples in the axial vorticity profile are due to the measurement noise. The vorticity of the vortex core region is still stronger than other radial positions at that cross-sectional plane, but less pronounced compared to the vorticity at $z / D=0.963$ and with $25 \%$ port closure. The maximum value of the axial vorticity has reduced to nearly half of the maximum value at $z / D=0.963$ for $25 \%$ intake port closure.

The mean axial vorticity distribution at $z / D=2.016$ and $z / D=3.068$ (Fig. 7f, i) respectively, is more uniformly distributed. This indicates that the initial strength of the vortex core decreases along the flow downstream by transferring vorticity to other weak vortical regions.

The turbulent kinetic energy increases further with the increase in the partial closure of the intake port from 25 to $50 \%$ (cf. Fig. 9b). The vortex core region now has a very low TKE value. The maximum value of TKE is observed in the region $X / R=0.4$ to 0.6 . This indicates that the overall velocity variance in the center of the jet-like axial velocity 

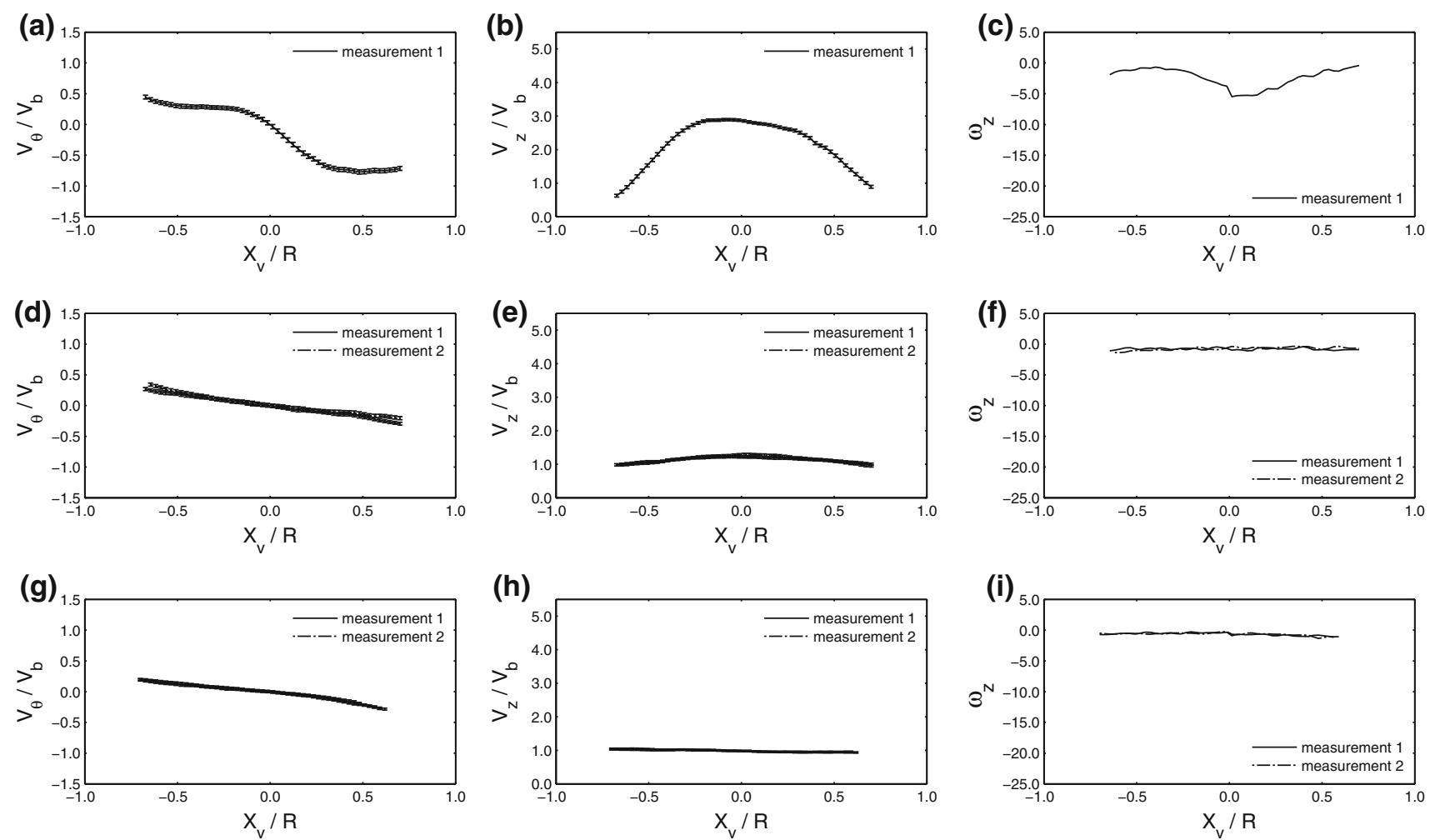

Fig. 7 Normalized time averaged profiles with $50 \%$ closed ports: a tangential velocity profile at $z / D=0.963$; $\mathbf{b}$ axial velocity profile at $z / D=0.963 ; \mathbf{c}$ axial vorticity profile at $z / D=0.963 ; \mathbf{d}$ tangential velocity profile at $z / D=2.016 ; \quad$ e axial velocity profile at

is very low. The asymmetric distribution of TKE in the vortex core region can be understood from an asymmetric jet-like profile of mean axial velocity component.

\subsection{Ports closed by $75 \%$}

The tangential velocity exhibits a solid body rotation throughout the cylinder, i.e., peak $v_{\theta}$ in the entire cylinder is shifted to the near wall positions (cf. Fig. 8a, d, g). The overall tangent velocity magnitude decays downstream of the flow.

At $z / D=0.963$ the axial velocity component $v_{z}$ still has a jet-like profile (Fig. 8b). However, the profile has a sharp peak compared to the $50 \%$ intake port closure (Fig. 7b). A significant change in the $v_{z}$ profile is observed at $z / D=$ 1.489, where $v_{z}$ changes back from jet-like to wake-like profile (Fig. 8e), which is characteristic of a vortex breakdown [13]. The profile for $v_{z}$ remains the same, i.e., wake-like at all downstream positions (Fig. 8h); however, the peak value $v_{z}$ is observed at large radial distance from the vortex center, indicating an increase in the vortex core size downstream of the flow.

When the intake port is closed by $75 \%$ (Fig. 8c), compared to the other positions of the piston, at $z / D=0.963$ $z / D=2.016 ; \mathbf{f}$ axial vorticity profile at $z / D=2.016 ; \mathbf{g}$ tangential velocity profile at $z / D=3.068 ; \mathbf{h}$ axial velocity profile at $z / D=3.068 ; \mathbf{i}$ axial vorticity profile at $z / D=3.068$

the strong vorticity of the vortex the core region no longer exists. Instead, the mean velocity field has nearly uniform $\omega_{z}$-distribution throughout the cylinder.

The increase in the value of the turbulent kinetic energy continues with the partial closure of the intake port. For $75 \%$, similar to $50 \%$, the vortex core region has a low value of TKE at $z / D=0.963$ (Fig. 9c). The distribution of TKE in the core region is comparatively more symmetric than at $50 \%$. This can be observed from the $v_{z}$ profile (Fig. 8b), which is more symmetric than the $v_{z}$ profile at $50 \%$ port closure (Fig. $7 \mathrm{~b}$ ).

\section{Conclusion}

The magnitudes of the tangential and axial velocities decay downstream due to friction with the internal cylinder wall. The tangential velocity profile, with a fully open port, is similar to a Burgers vortex, i.e., an inner forced-vortex core and free-vortex outer region. The higher velocities are observed at some intermediate radial position between the cylinder wall and the geometric center, where force and free vortex regions meet. With the downstream decay in swirl, the size of the forced vortex region increases. 

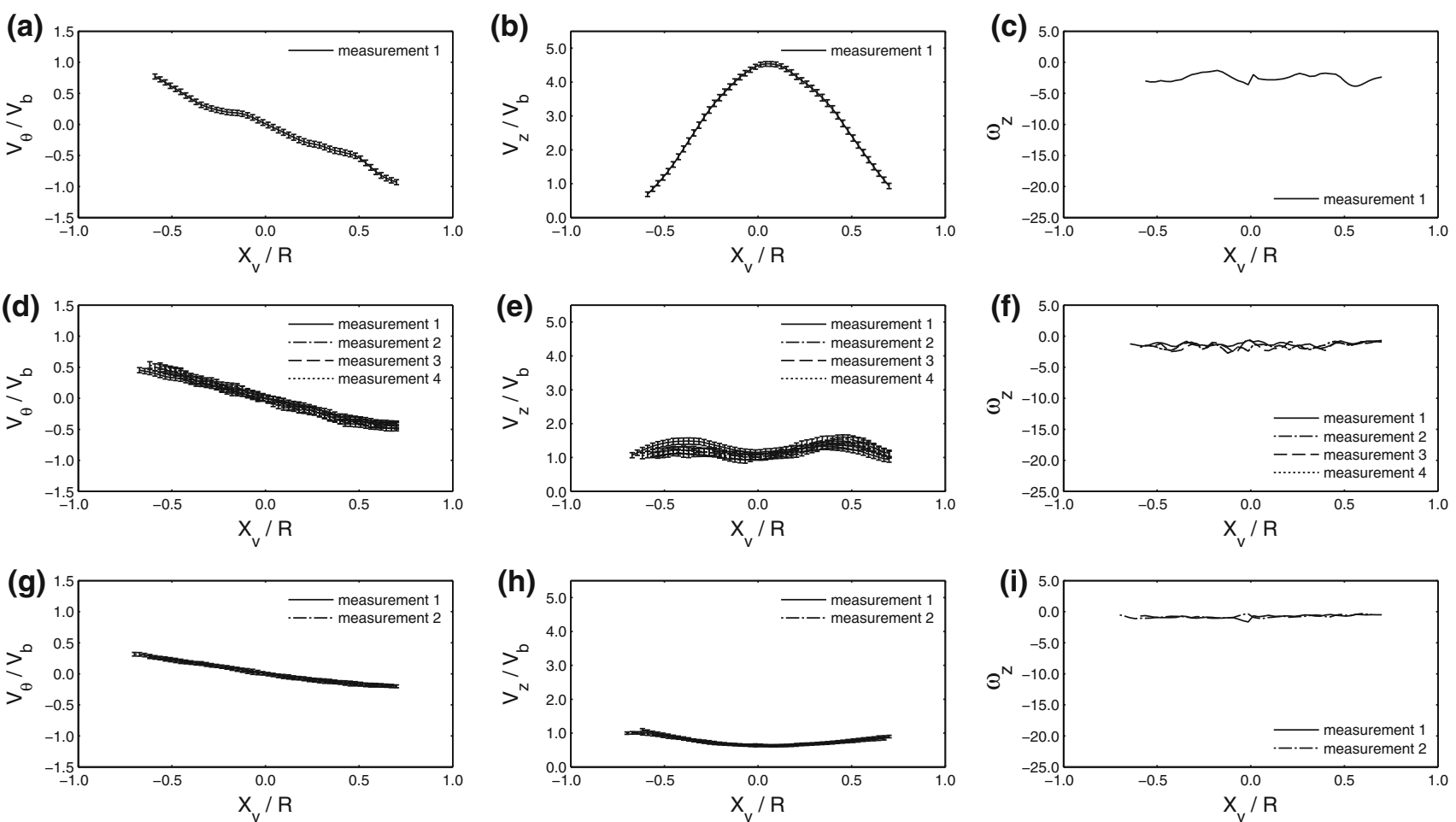

Fig. 8 Normalized time averaged profiles with $75 \%$ closed ports: a tangential velocity profile at $z / D=0.963 ; \mathbf{b}$ axial velocity profile at $z / D=0.963$; c axial vorticity profile at $z / D=0.963$; d tangential velocity profile at $z / D=1.489$; e axial velocity profile at

At piston positions partially closing the port, the tangential velocity profile starts changing to a forced vortex, i.e., higher velocities are observed near the cylinder walls. This change in the velocity profile begins from cross-sectional positions near the cylinder outlet and moves to upstream positions as the piston gradually closes the port. However, in the case of piston positions with $50 \%$ port closure, the tangential velocity profiles resembled a wall-jet-like profile rather than the forced vortex. For the cross-sectional positions close to the intake port, the partial closure of the intake port introduces asymmetry and variation in the mean tangential velocity profile.

Fully open ports result in an axial velocity with wakelike profile at all measuring planes. However, no reverse flow at the vortex core has been observed. The downstream decay in swirl is decreasing the wake effect by transferring more mass into the wake region. As the piston starts closing the ports, the axial velocity profile changes from wake-like to jet-like at $50 \%$ port closure. However, at $75 \%$ port closure the jet-like mean axial velocity profile at the crosssectional position close to the intake port changes back to wake-like at the adjacent downstream cross-sectional position. This is characteristic of a vortex breakdown. The mean axial velocity profile then continues to have the wakelike profile at the remaining downstream positions. $z / D=1.489 ; \mathbf{f}$ axial vorticity profile at $z / D=1.489 ; \mathbf{g}$ tangential velocity profile at $z / D=3.068 ; \mathbf{h}$ axial velocity profile at $z / D=3.068 ; \mathbf{i}$ axial vorticity profile at $z / D=3.068$

The mean axial vorticity of the mean velocity field has a Gaussian-like profile when the intake port is fully open. However, with the downstream decay of swirl as well as the partial closure of the intake port, the vorticity confined in the vortex core region is transmitted to the outer regions. This results in a comparatively uniform mean axial vorticity distribution throughout the cylinder for the $75 \%$ port closure. The turbulent kinetic energy increases with the partial closure of the intake port. For a given crosssectional plane, the distribution of TKE seems to be understood from the mean axial velocity distribution. For a wake-like $v_{z}$ profile, the maximum value of TKE is observed in the vortex core region and the minimum value is observed in the high $v_{z}$ region. Similarly, for a jet-like $v_{z}$ profile, the minimum value is observed in the center of the jet and maximum value is observed in the jet skirt region. The experimental results presented in this paper provides insight into the nature of the in-cylinder confined swirling flow during a uniflow scavenging process. With the piston motion, the change in the tangential and axial velocity profiles, both at different piston position and at different cross-sectional planes, represent a specific type of vortex. Studying the characteristics of the different types of vortex structures is a key to the understanding of the real engine scavenging process, including critical issues such as the 

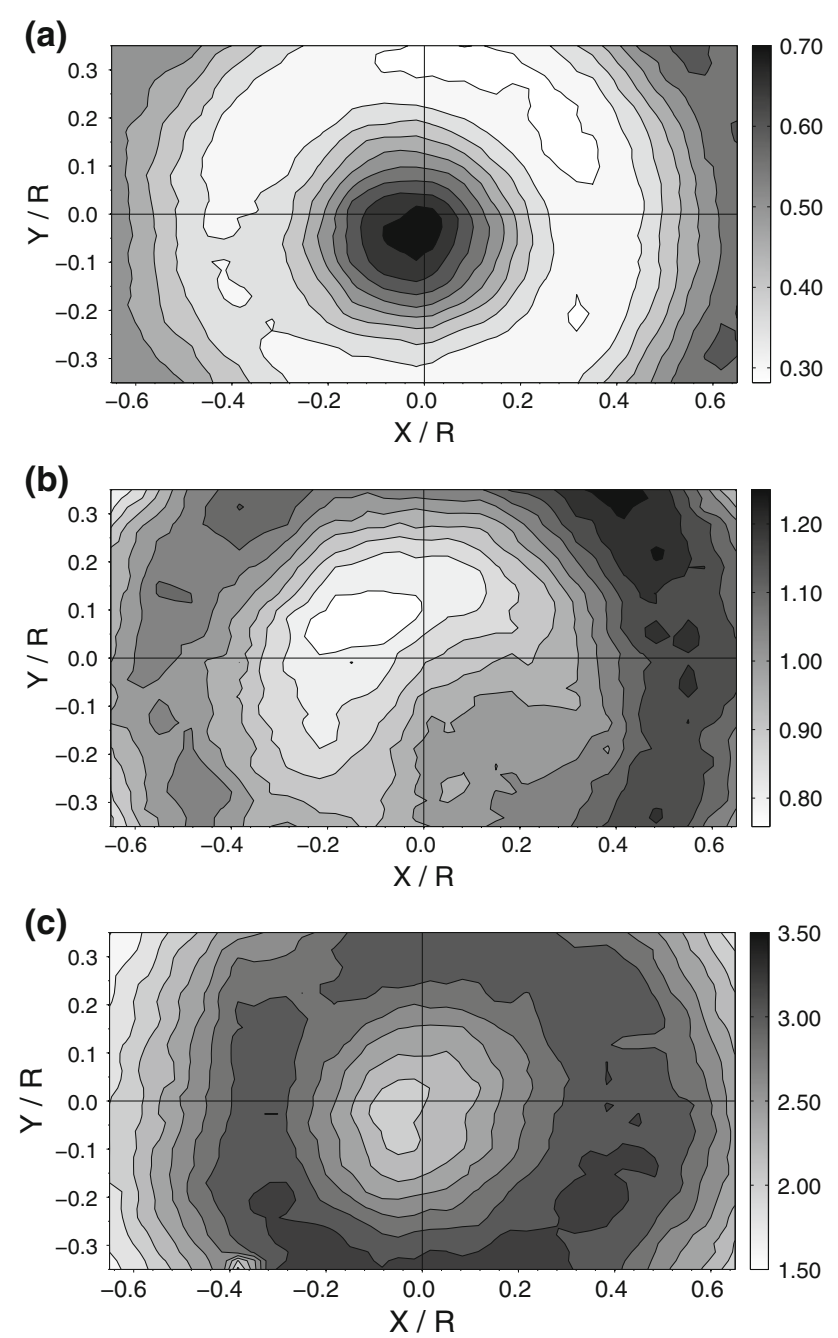

Fig. 9 Time average turbulent kinetic energy at $z / D=0.963$ for the a $25 \%$, b $50 \%$ and c $75 \%$ closed ports

mixing of fresh charge with exhaust gases. Moreover, the modelling of swirling flow is a challenge to standard computational fluid dynamics (CFD) tools, and the present, fixed-piston experiment provides an essential benchmark for the validation of CFD tools for engine simulations.

Acknowledgments The authors acknowledge support from MAN Diesel \& Turbo, the Danish Centre for Maritime Technology (DCMT), and the Danish Research Council of Independent Research (Grant 09-070608).The authors furthermore acknowledge CIMAC for granting permission to publish some of the results presented by the authors at the CIMAC 2010 Congress.

\section{References}

1. Pevzner LA (1998) Aspects of marine low-speed, cross-head diesel engine lubrication. Lubr Eng 54(6):16-21

2. Nakagawa $H$, Kato $S$, Tateishi $M$, Adachi $T$, Tsujimura $H$, Nakashima M (1990) Airflow in the cylinder of a 2-stoke cycle uniform scavenging diesel engine during compression stroke. Jpn Soc Mech Eng 33(3):591-598

3. Litke B (1999) The influence of inlet angles in inlet ports on the scavenving process in two-stroke uniflow-scavenged engine. Mar Tech III 45:247-252

4. Nishimoto K, Takeyuki K (1984) A study on the influence of inlet angle and Reynolds number on the flow-pattern of uniflow scavenging air. SAE Tech Paper Ser 93

5. Dedeoglu N (1988) Improvement of mixture formation in a uniflow-scavenged two-stroke engine. SAE Tech Paper Ser 901536

6. DantecDynamics (2009) Dantec dynamicstudio v 2.3 user manual. Tech rep

7. Gupta AK, Lilley DG, Syred N (1984) Swirl flows. Abacus Press, Tunbridge Wells

8. Alekseenko SV, Kuibin PA, Okulov VL (2007) Theory of concentrated vortices: an introduction. Springer, Berlin

9. Lam H (1993) An experimental investigation and dimensional analysis of confined vortex flows. PhD thesis, Concordia University, Quebec (unpublished)

10. Steenbergen W, Voskamp J (1998) The rate of decay of swirl in turbulent pipe flow. Flow Meas Instrum 9(2):67-78

11. Pani BS, Rajaratnam N (1976) Swirling circular turbulent wall jets. J Hydraul Res 14(2):145-154

12. Rajaratnam N (1976) Turbulent jets. Elsevier, Amsterdam

13. Escudier MP, Bornstein J, Maxworthy T (1982) The dynamics of confined vortices. Proc R Soc Lond A 382:335-350 